\title{
Rapid Liquefaction of Wood in Polyhydric Alcohols Under Microwave Heating and its Liquefied Products for Preparation of Rigid Polyurethane Foam
}

\author{
Zhifeng Zheng ${ }^{*}, 1$, Hui Pan ${ }^{2}$, Yuanbo Huang ${ }^{1}$, Y. Hse Chung ${ }^{3}$, Xuliang Zhang ${ }^{1}$ and Hao Feng ${ }^{1}$ \\ ${ }^{I}$ Key Laboratory of Forest Resources Conservation and Use in the Southwest Mountains of China (SWFU), Ministry of \\ Education, Southwest Forestry University, Kunming 650224, China \\ ${ }^{2}$ Calhoun Research Station, Louisiana State University AgCenter, Calhoun, Louisiana 71225, USA \\ ${ }^{3}$ Southern Research Station, USDA Forest Service, Pineville, Louisiana 71360, USA
}

\begin{abstract}
Comparing to the conventional bath heating, the effects of liquefaction parameters on the degree of liquefaction of wood in polyhydric alcohols by using microwave as an energy source were evaluated. Polyurethane foams were successfully prepared from the liquefied biomass-based polyols. The effects of heating methods (conventional bath heating and microwave) and liquefaction reaction time on the properties of liquefied biomass-based polyols were investigated. The influences of isocyanate index on the properties of rigid polyurethane foams were also discussed, and feasible formulations for preparing liquefied biomass-based polyurethane foams were proposed. The results indicated that rapid liquefaction of wood in polyhydric alcohols under microwave heating could be achieved. The foams prepared from liquefied biomass-based polyols obtained by microwave-assistant liquefaction exhibited excellent mechanical properties.
\end{abstract}

Keywords: Polyurethane rigid foam, liquefaction, southern pine, microwave heating.

\section{INTRODUCTION}

Polyurethane foams (PU Foams) are used widely in many fields as heat-insulating, packaging, structural, flotation and load bearing materials, due to their ease of processing and unique combination of excellent thermal insulation and mechanical properties. Traditional PU foams are prepared by the polymerization of polyol with isocyanate simultaneously occurring with gas generation resulting from the chemical reaction of water of blowing agent evaporation by the exothermic reaction itself. However, the raw materials for preparing PU foams, based on polyol (polyether of polyester polyols) and isocyanate, are obtained mainly from fossil resources. With increasing concern of the shortages of fossil resources and the impetus for reducing costs of polyurethane, preparation of polyols from renewable biomass resources has been an interesting subject in the polyurethane industry. Biomass as natural polymer contains more than one hydroxyl group in the molecular chains, and can be used as polyol for preparing polyurethane materials. By the hydroxyalkylation reaction, polyols with desirable hydroxyl values and viscosities have been prepared from many hydroxyl-containing biomass substances such as sorbitol [1], sucrose [2], methyl glucoside [3], lignins [4, 5], and so forth. These polyols have become important ingredients for rigid polyurethane foams because of the great functionalities of the starting materials.

*Address correspondence to this author at the Faculty of Wood Science and Technology, Southwest Forestry University, Bailongsi 300, Kunming 650224, China; Tel: +86-871-3863609; Fax: +86-871-3863989;

E-mails: zhengzhifeng@swfu.edu.cn, zhengzhifeng666@hotmail.com
There were also many reports concerning direct incorporation of biomass resources such as starch, lignocellulosics, lignins, coffee grounds, bark of trees, and so forth into polyurethane formulations [6-8]. It was reported by Hatakeyama and co-workers that rigid polyurethane foams containing as much as $50 \%$ lignocellulosics or other biomass could be obtained by mixing the biomass with liquid polyols before foaming. However, except for a few cases, the biomass was insoluble in the polyols. In these cases, the biomass could only act as solid fillers rather than as reactive components in the foam structures, and as a result, foams with high densities were apt to be obtained.

Recently, liquefaction of biomass in the presence of some organic solvents has been developed [9]. The liquefied products of biomass prepared in the presence of alcohols are rich in hydroxyl groups. One of the promise applications for these liquefied products is to prepare polyurethanes (PU) materials. It has been found that if polyhydric alcohols with appropriate molecular weights were used as reagents in the liquefaction of biomass, the resulting liquefied mixtures can be used directly as polyols to prepare polyurethane foams without any additional reaction of treatment [10-13].

However, almost the liquefaction reactions of biomass in organic solvents have been carried out with the conventional convective-conductive heating sources such as water, oil, salt bath, fluidized sand bath and electrical furnaces. These methods, however, are relatively slow and inefficient for transferring energy into the system, because they depend on the thermal conductivities of materials. In contrast, microwave irradiation produces efficient internal heating or in-core volumetric heating by direct coupling of microwave energy with the molecules that may be the solvents, reagents 
or catalysts that are present in the reaction mixture. The main advantage of microwaves over conventional heating sources is that the irradiation penetrates and simultaneously heats the bulk of the material.

In this study, many attempts have been made using the liquefaction technology with microwave-assistant to introduce wood into polyols for preparation of PU foams. The effects of liquefaction parameters on the liquefaction and properties of biomass-based polyols, the formulations and characteristics of PU foams were investigated.

\section{EXPERIMENTAL}

\subsection{Materials}

Wood meal (southern pine, Pinus sp., 40 to 60 mesh) was dried in an oven at $105^{\circ} \mathrm{C}$ for 24 hours before use. Polyethylene glycol \#400 (PEG \#400, Mw=400), glycerin, sulfuric acid, phosphoric acid and sodium hydroxide were of reagent grades and were obtained from commercial sources. Other ingredients included diphelylmethane diisocyanate (MDI) (MR100, by Huntsman ICI Chemicals LLC Ind. Ltd., U.S.A.), dibutyltin dilaurate (DBTDL) (Katsuta Ind. Ltd., Japan), and silicone surfactant SH193 (by Dow Corning Corporation, U.S.A.) were used as received.

\subsection{Preparation of the Liquefied Biomass-Based Polyols}

The liquefaction reagent was mixture of PEG \#400 and glycerin. Sulfuric acid was used as a catalyst. The liquefactions were carried out in a microwave extraction system (Ethos EX, Milestone) equipped with an independent controller (Model 640 Terminal) and a temperature sensor that can be inserted directly into the sealed $100 \mathrm{~mL}$ teflon reaction vessels. A typical workup procedure for the experiments was as follows. 2 gram of the wood sample and 14 gram of the PEG 400/glycerin (70/30, w/w) which contains $3 \%$ sulfuric acid as a catalyst were load into the teflon vessel, then premixed them together by stirring thoroughly before liquefaction. The normal heating program used was $7 \mathrm{~min}$ at $800 \mathrm{~W}$. After the desired additional liquefaction time, the pressurized vessel was immersed in cold water and could safely be opened after $15 \mathrm{~min}$. Twenty vessels of the samples were put together. An equivalent amount of sodium hydroxide aqueous solution (50\%) was added to neutralize the sulfuric acid catalyst, thus the liquefied biomass-based polyols were obtained. In order to compare, the liquefaction was also carried out by using bath oil heating.

\subsection{Measurements of the Unliquefied Residues of Biomass}

About $1 \mathrm{~g}$ of the liquefied mixture was dissolved in $20 \mathrm{ml}$ of methanol solvent, stirred for more than 4 hours, and then the dilution was filtrated through glass filter paper under reduced pressure. The solid residues were dried in an oven at $105^{\circ} \mathrm{C}$ to a constant weight, and the the residue percent of the biomass (the residual contents in the liquefied biomassbased polyols) was calculated by the following equation (1):

$R(\%)=\left(m_{1} / m_{0}\right) \times 100$

where $R$ is the residual rate in percent (\%), $m_{1}$ is oven-dry weight of the solid wood residue $(\mathrm{g}), m_{0}$ is oven-dry weight of the starting wood meal $(\mathrm{g})$.
2.4. Measurements of the Specific Gravity and Apparent Viscosities of the Liquefied Biomass-Based Polyols

The specific gravity of the liquefied biomass-based polyols was measured according to ASTM D 4669. The apparent viscosities of the liquefied biomass-based polyols were measured by a Viscosity Centipoise 98936 series viscometer according to ASTM D 4878. Measurements were conducted at $25 \pm 0.1^{\circ} \mathrm{C}$.

\subsection{Measurements of the Acid Number of the Liquefied Biomass-Based Polyols}

The acid number of the biomass-based polyol was measured by the titration method according to ASTM D 4662-08. The number of milligrams of $\mathrm{KOH}$ required to neutralize the acid in one gram of sample was calculated as follows (Eq.2):

Acid number $(\mathrm{mg} \mathrm{KOH} / \mathrm{g})=\frac{56.1 \times V \times N}{W}$

where $V$ is the consumed amount of potassium hydroxide solution at the neutralization point $(\mathrm{ml}) ; N$ is the equivalent concentration of potassium hydroxide solution used; and $W$ is the weight of the sample $(\mathrm{g})$.

\subsection{Measurements of the Hydroxyl Number of the Liquefied Biomass-Based Polyols}

The hydroxyl number of a liquefied mixture was measured as the number of milligram of potassium hydroxide equivalent to the phthalic anhydride consumed in the phthalification of one gram of sample according to ASTM D 4274-05. The hydroxyl number was corrected and calculated according to the following equation (3):

Hydroxyl number

$(\mathrm{mg} \mathrm{KOH} / \mathrm{g})=[(B-C) \times N \times 56.1] / W+A$

where $B$ is the blank titration volume of the sodium hydroxide solution for the phthalic anhydride-pyridine reagent $(\mathrm{ml}) ; C$ is the volume of the sodium hydroxide solution consumed in the back-titration of the sample (ml); $N$ is the equivalent concentration of $\mathrm{NaOH}$ solution; $W$ is the weight of the sample $(\mathrm{g})$; and $A$ is the acidity of the sample (mg KOH/g).

\subsection{FTIR Analysis of the Liquefied Biomass-Based Polyols}

The changes of components of the liquefied biomassbased polyols during the liquefaction were analyzed by using a Fourier transform infrared (FTIR) spectrometer. Infrared spectra were measured with a Nicolet MAGNA-IR 560 spectrometer.

\subsection{Preparation of the Rigid Foams}

The $\mathrm{pH}$ of the biomass-based polyol obtained above was adjusted by adding $50 \mathrm{wt} \%$ sodium hydroxide aqueous solution. Thus, the definite amounts of liquefied biomassbased polyol, catalyst, surfactant, and water were premixed thoroughly in a plastics cup. Then, the prescript amount of MDI (at an isocyanate index of 80 to 120 ) was added and mixed quickly at a high stirring speed of $8,000 \mathrm{rpm}$ for $10-15$ seconds. The mixture then was poured immediately into a 
$17 \times 13 \times 13 \mathrm{~cm}$ cardboard box and was allowed to rise freely at room conditions. Foam was removed from the box after one hour and was allowed to cure at room temperature for two days before cutting into test samples.

\subsection{Measurements of Properties of the Rigid Foams}

The apparent density of the rigid foam was measured according to ASTM D1622-08. Measurements of mechanical properties of the foams are based on the ASTM D1621-04a. The foams were cut into $50 \mathrm{~mm} \times 50 \mathrm{~mm} \times 50 \mathrm{~mm}$ specimens. The specimens were conditioned for 48 hours at $23^{\circ} \mathrm{C}$ and $50 \%$ relative humidity and then were callipered and weighted to determine apparent densities. The compressive properties of the foams then were measured by an INSTRON 4465 electronic mechanical tester. The measurements were made in the direction perpendicular to the foam rise at a constant crosshead rate speed of 5 $\mathrm{mm} / \mathrm{min}$. The compressive strengths of the foams were determined as the stresses at the yield points of the specimen deformation in the case of specimens showing yield points approximately $13 \%$ strain, otherwise the strengths were determined as the stresses at $10 \%$ strain. At least five specimens were measured for every foam formulation.

\section{RESULTS AND DISCUSSION}

\subsection{Effect of Reaction Conditions on the Liquefaction}

\subsubsection{Effect of Reaction Time on the Liquefaction}

Fig. (1) presents the effect of reaction time on the liquefaction of wood in polyhydric alcohols under conventional bath heating and microwave heating. The residue percent descended rapidly to about $0 \%$ within 2 min under microwave heating, though it was $78.82 \%$ within 2 min under conventional bath heating. It revealed that the microwave heating had a higher efficiency of liquefaction than the conventional bath heating before $30 \mathrm{~min}$. The microwave can heat the stuff in vessel in a short time and make degradation of biomass very quickly, resulting that the residue percent decreased rapidly.

\subsubsection{Effect of Temperature on the Liquefaction}

Fig. (2) shows the effect of temperature on the liquefaction of wood in polyhydric alcohols under microwave heating. In $30 \mathrm{~min}$, the liquefaction effect was improved when the temperature increased from 90 to 130 ${ }^{\circ} \mathrm{C}$. The residue percent is $10.13 \%$ as the temperature of 90 ${ }^{\circ} \mathrm{C}$. But it could not achieve the same liquefaction effect under the same bath heating temperature [14]. It indicated that microwave heating had a higher efficiency of liquefaction than conventional bath heating at low reaction temperature. When the temperature increased to $170{ }^{\circ} \mathrm{C}$, repolymerization of liquefied products took precedence and the residue percent had a little increase. So, the reaction temperature should not be excessively high and $150{ }^{\circ} \mathrm{C}$ was enough.

\subsubsection{Effect of Solvent/Wood Weight Ratio on the Liquefaction}

Fig. (3) presents the effect of solvent/wood weight ratio on the liquefaction in polyhydric alcohols under microwave heating. The role of solvent is to dissolve the liquefied products and prevent them from re-polymerizing. As shown in Fig. (3), the solvent/wood weight ratio showed a little effect on the liquefaction under microwave heating. It is different from the bath heating [14]. But low solvent/wood ratio made the plastic rotor stirring difficult in the vessel in the microwave heating system. So, the solvent/wood weight ratio of 7 in the microwave heating system would be suitable.

\subsubsection{Effect of Moisture Content of Wood on the Liquefaction}

Hydrolysis reaction is also an important reaction in the liquefaction of wood in polyhydric alcohols, especially under

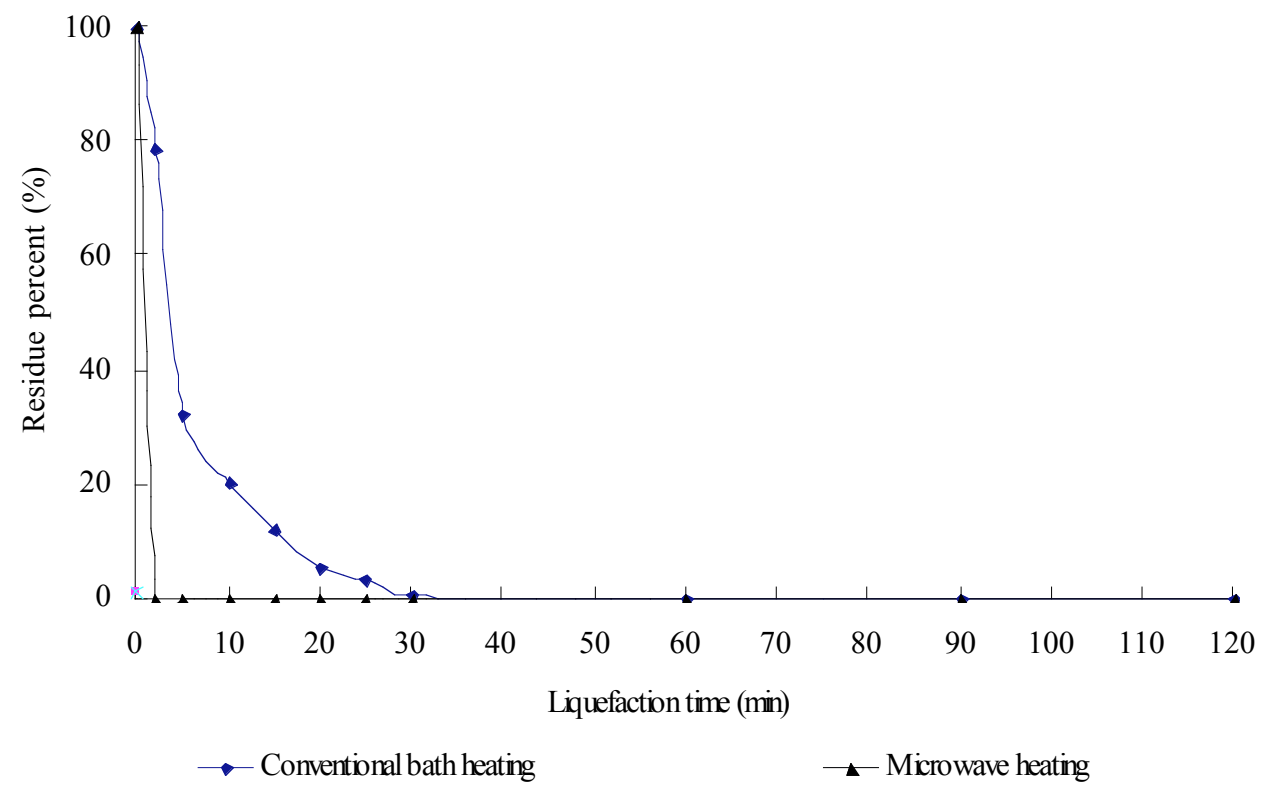

Fig. (1). Effect of reaction time on the liquefaction of wood in polyhydric alcohols. (Conditions: PEG 400/glycerin=7/3, sulfuric acid concentration $=3 \%$, solvent $/$ wood weight ratio $=7$, moisture content of wood $=0 \%, 150^{\circ} \mathrm{C}$ ). 


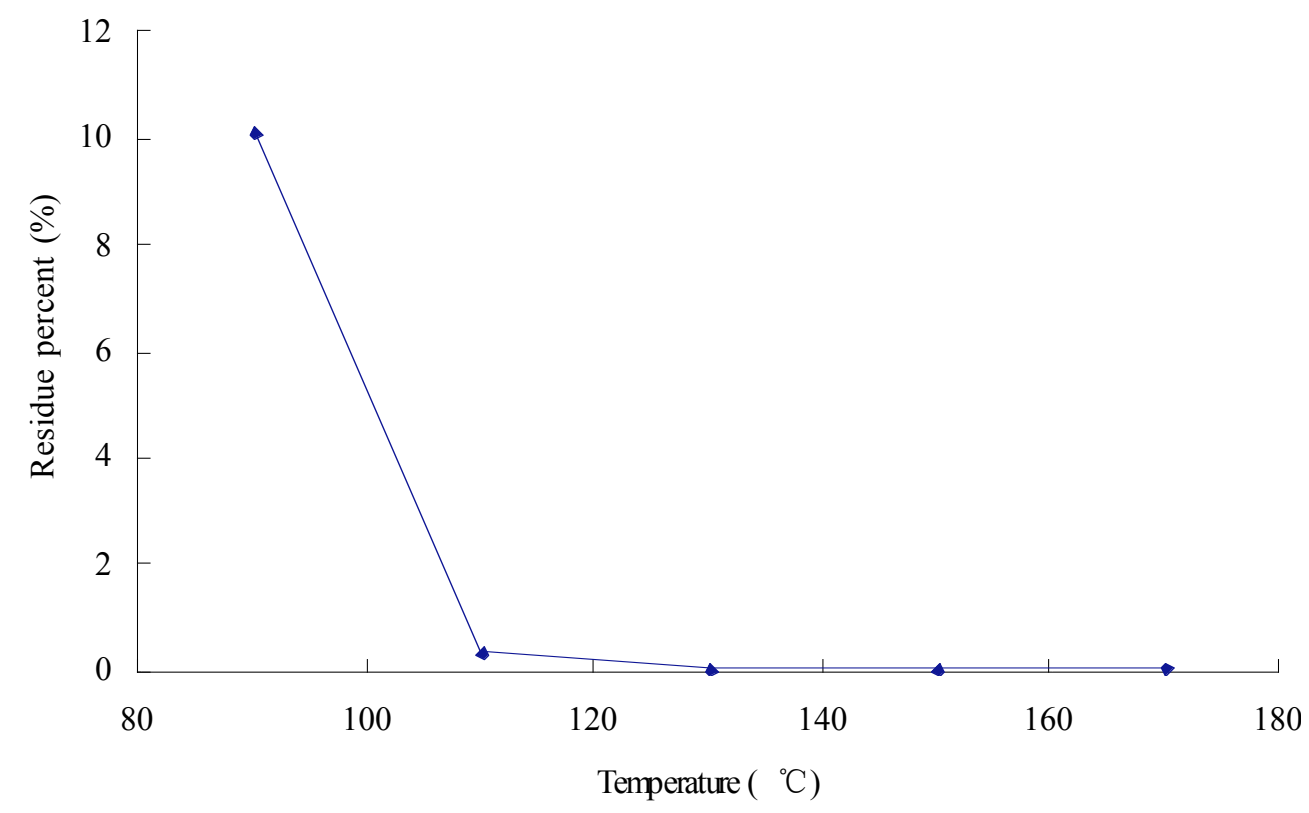

Fig. (2). Effect of reaction temperature on the liquefaction of wood in polyhydric alcohols under microwave heating. (Conditions: PEG 400/glycerin $=7 / 3$, sulfuric acid concentration $=3 \%$, solvent $/$ wood weight ratio $=7$, moisture content of wood $=0 \%, 30 \mathrm{~min}$ ).

microwave heating. In order to study the influence of hydrolysis on the liquefaction under microwave heating, wood samples at different moisture content were used. Fig. (4) shows the effect of moisture content of wood on the liquefaction. Existence of water in the system slowed down the liquefaction of wood. This might be due to the lessening of reaction temperature and solvent with excessive water.

\subsubsection{Effect of Catalyst Concentration on the Liquefaction}

In the experiment, sulfuric acid was added as the catalyst of the liquefaction reaction from $0.5 \%$ to $3 \%$. The residue percent was $89.51 \%$ without catalyst under microwave heating. But when the concentration of sulfuric acid rose to
$0.5 \%$, the residue percent showed a big descent to $0.06 \%$. It indicated that a small amount of sulfuric acid could show an excellent efficiency of liquefaction under microwave heating. However, high concentration of sulfuric acid had strong oxidizability and may cause other secondary reactions. So, $0.5 \%$ sulfuric acid is recommended in practical production.

\subsection{Properties of the Liquefied Biomass-Based Polyols}

Table 1 shows the properties of the liquefied biomassbased polyols obtained from microwave -assistant liquefaction by using sulfuric acid as the catalyst. There was not any residue in the liquefied mixture. So, the liquefied

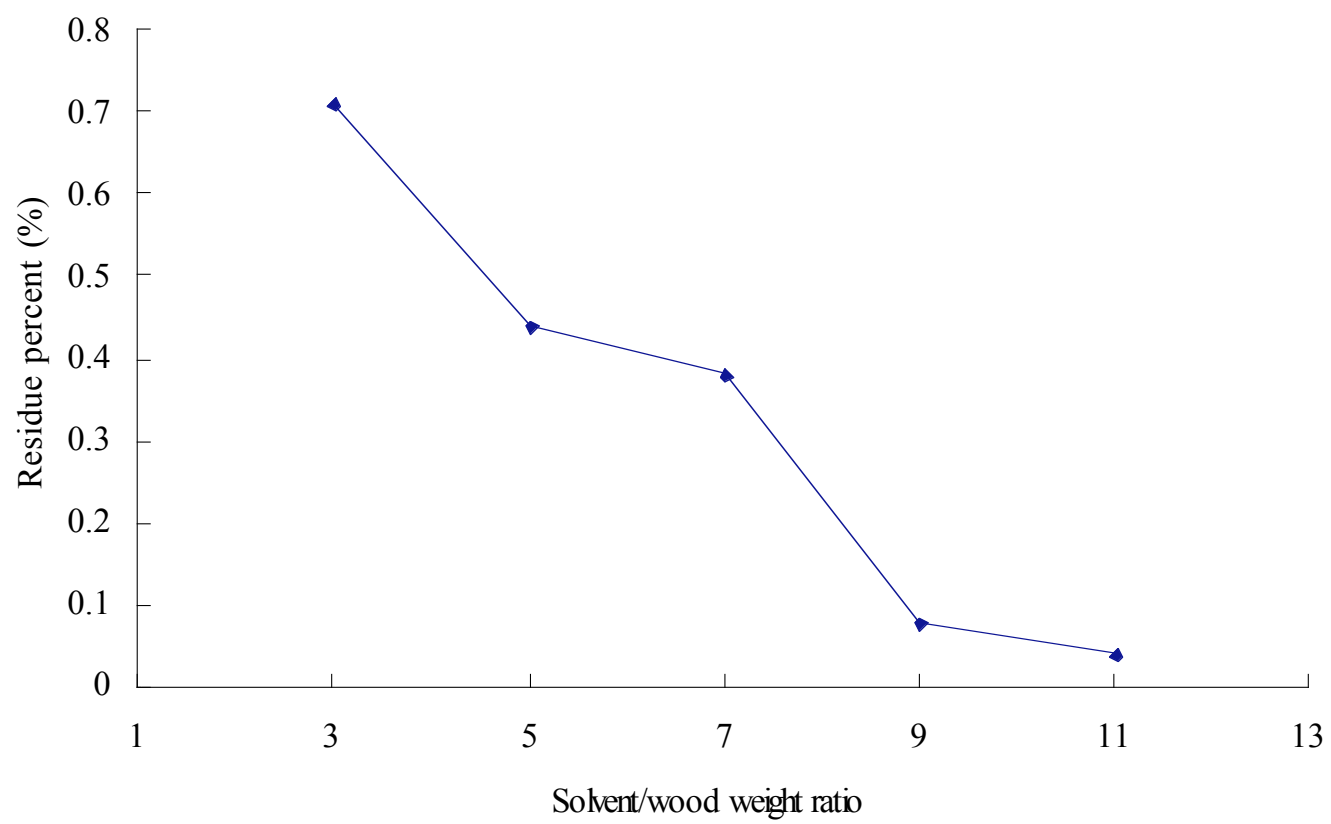

Fig. (3). Effect of solvent/wood weight ratio on the liquefaction of wood in polyhydric alcohols under microwave heating. (Conditions: PEG 400 /glycerin $=7 / 3$, sulfuric acid concentration $=3 \%$, moisture content of wood $=0 \%, 30 \mathrm{~min}, 150^{\circ} \mathrm{C}$ ). 


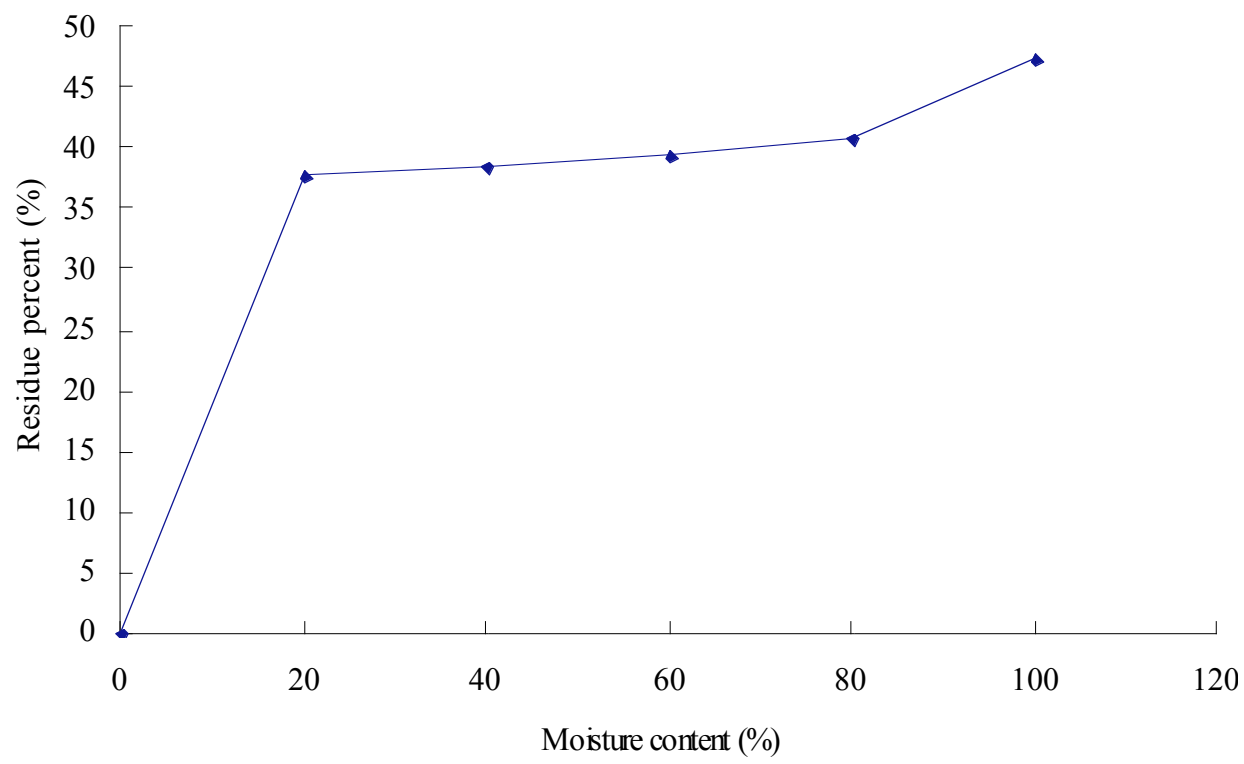

Fig. (4). Effect of moisture content of wood on the liquefaction in polyhydric alcohols under microwave heating. (Conditions: $\mathrm{PEG}$ $400 /$ glycerin $=7 / 3$, sulfuric acid concentration $=3 \%$, solvent $/$ wood weight ratio $=7,30 \mathrm{~min}, 150^{\circ} \mathrm{C}$ ).

biomass-based polyols can be directly to use for preparing rigid PU foams.

As for the specific gravity of the liquefied mixture obtained by using sulfuric acid as the catalyst, it is higher than PEG \#400, but lower than the mixture of PEG \#400 and glycerin.

It has been reported that the viscosity of the mixture obtained by traditional heating is as high as $270,000 \mathrm{Cp}$ [15]. Compared to the traditional bath heating liquefaction, the viscosity of the liquefied mixture obtained by microwaveassistant liquefaction is lower by using the same acid as the catalyst, due to no wood solid residue remaining in the liquefied mixture. These values listed in Table $\mathbf{1}$ are somewhat larger than those of the fossil-based polyols, but are still suitable for the preparation of polyurethane foam.

From the Table 1, the most significant change is the hydroxyl value (about half of the mixture of PEG \#400 and glycerin), especially in the initial period of the microwaveassistant liquefaction. Table $\mathbf{1}$ also shows that the hydroxyl value of the liquefied mixture decreases significantly and acidic substances are produced with increasing liquefaction time. This result suggests that apart from the alcoholysis reaction, dehydration and/or oxidation reactions also occur during the liquefaction.

The FTIR technique was employed to investigate the functional groups of liquefied biomass-based polyols in the liquefaction. Figs. $(\mathbf{5}, \mathbf{6})$ shows FTIR spectra of the liquefied biomass-based polyols under microwave heating and conventional bath heating, respectively. The IR absorbance peak of the aromatic ring appeared at $1454 \mathrm{~cm}^{-1}, 1652 \mathrm{~cm}^{-1}$ and $843 \mathrm{~cm}^{-1}$ in both samples from the liquefaction under conventional bath heating and microwave heating. It revealed that lignin was easy to be degraded at the initial stage of the liquefaction and dissolved in the polyhydric alcohols, whatever the heating way is. The presence of hydroxyl group in the liquefied biomass-based polyol from the liquefaction under microwave heating, was reflected by the transmittance peaks at wavenumbers of $3385 \mathrm{~cm}^{-1}$ and $1028 \mathrm{~cm}^{-1}$ (due to $-\mathrm{OH}$ in primary alcohol). The peak of the

Table 1. Properties of the Liquefied Biomass-Based Polyols

\begin{tabular}{|c|c|c|c|c|c|}
\hline \multicolumn{2}{|c|}{ Reaction Time [min] } & $\begin{array}{c}\text { Specific Gravity } \\
{\left[\mathrm{g} / \mathrm{cm}^{3}\right]}\end{array}$ & $\begin{array}{c}\text { Apparent Viscosity } \\
{[\mathrm{cP}]}\end{array}$ & $\begin{array}{l}\text { Hydroxyl Value } \\
\text { [mg KOH/g] }\end{array}$ & $\begin{array}{l}\text { Acid Value } \\
{[\mathrm{mg} \mathrm{KOH} / \mathrm{g}]}\end{array}$ \\
\hline \multirow{4}{*}{ Microwave heating } & 5 & 1.13 & 1420 & 357 & 19.6 \\
\hline & 30 & 1.13 & 1450 & 323 & 27.8 \\
\hline & 60 & 1.13 & 1480 & 334 & 26.2 \\
\hline & 120 & 1.13 & 1720 & 291 & 26.1 \\
\hline \multirow{4}{*}{ Conventional bath heating } & 5 & 1.12 & 7880 & 622 & 18.8 \\
\hline & 30 & 1.13 & 2070 & 565 & 18.4 \\
\hline & 60 & 1.13 & 1920 & 523 & 19.8 \\
\hline & 120 & 1.13 & 1930 & 489 & 21.2 \\
\hline \multicolumn{2}{|l|}{ PEG \#400 } & 1.12 & 140 & 280 & - \\
\hline \multicolumn{2}{|l|}{ PEG \#400/glycerin } & 1.16 & 360 & 700 & - \\
\hline
\end{tabular}

Conditions: PEG 400/glycerol/sulfuric acid=70/30/3 (by weight), Wood/Solvent=1/7 (w/w), Reaction Temperature of $150^{\circ} \mathrm{C}$. 
liquefied biomass-based polyol from the liquefaction under microwave heating at wavenumbers of $1090 \mathrm{~cm}^{-1}$, which was the peak of $\mathrm{C}-\mathrm{O}$ bond stretching vibration from polysaccharide, was higher than that from the liquefaction under conventional bath heating at the same reaction time. It indicated that cellulose was easier to be degraded under microwave than bath heating. As all know, cellulose is difficult to be degraded in the liquefaction. But as shown in this study, microwave can apply great power in a short time and make cellulose degraded into low molecular weight compounds immediately. And there is no residue maintained in the liquefaction (as shown is Fig. 1). Totally, rapid liquefaction of wood in polyhydric alcohols by using microwave as the heating system can be achieved.

\subsection{Basic Foam Formulation for Biomass-Based Polyols and its Properties}

The liquefied mixtures obtained above were used directly to prepare polyurethane foam by reaction with methylene diphenyl diisocyanate (MDI). Table $\mathbf{2}$ is the foam formulations for the liquefied biomass-based polyols from the liquefaction under microwave. For improving the mixing

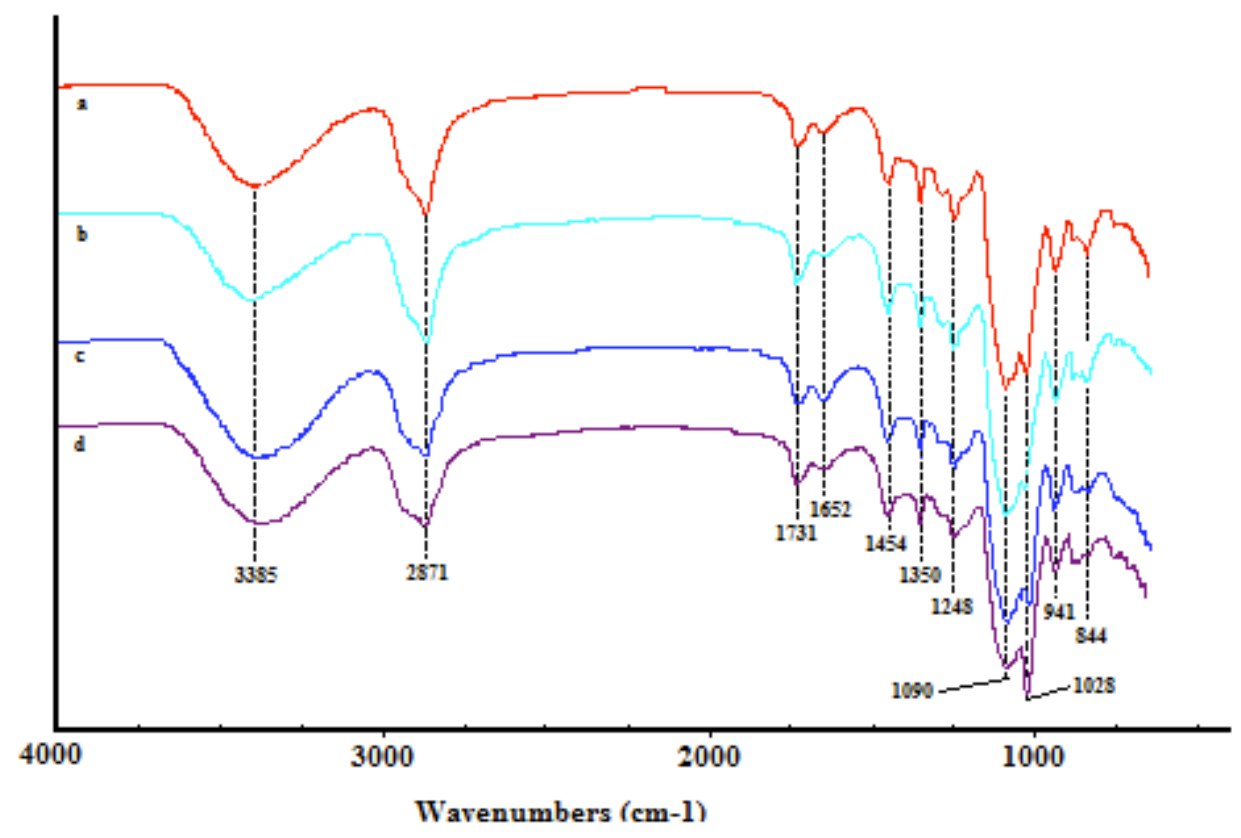

Fig. (5). FTIR spectra of the liquefied biomass-based polyols of wood under microwave heating. a. 5 min; b. 30 min; c. 60 min; d. 120 min. (Microwave heating conditions: $\mathrm{PEG} 400 /$ glycerin $=7 / 3$, sulfuric acid concentration $=3 \%$, solvent $/$ wood weight $\mathrm{ratio}=7,150^{\circ} \mathrm{C}$ ).

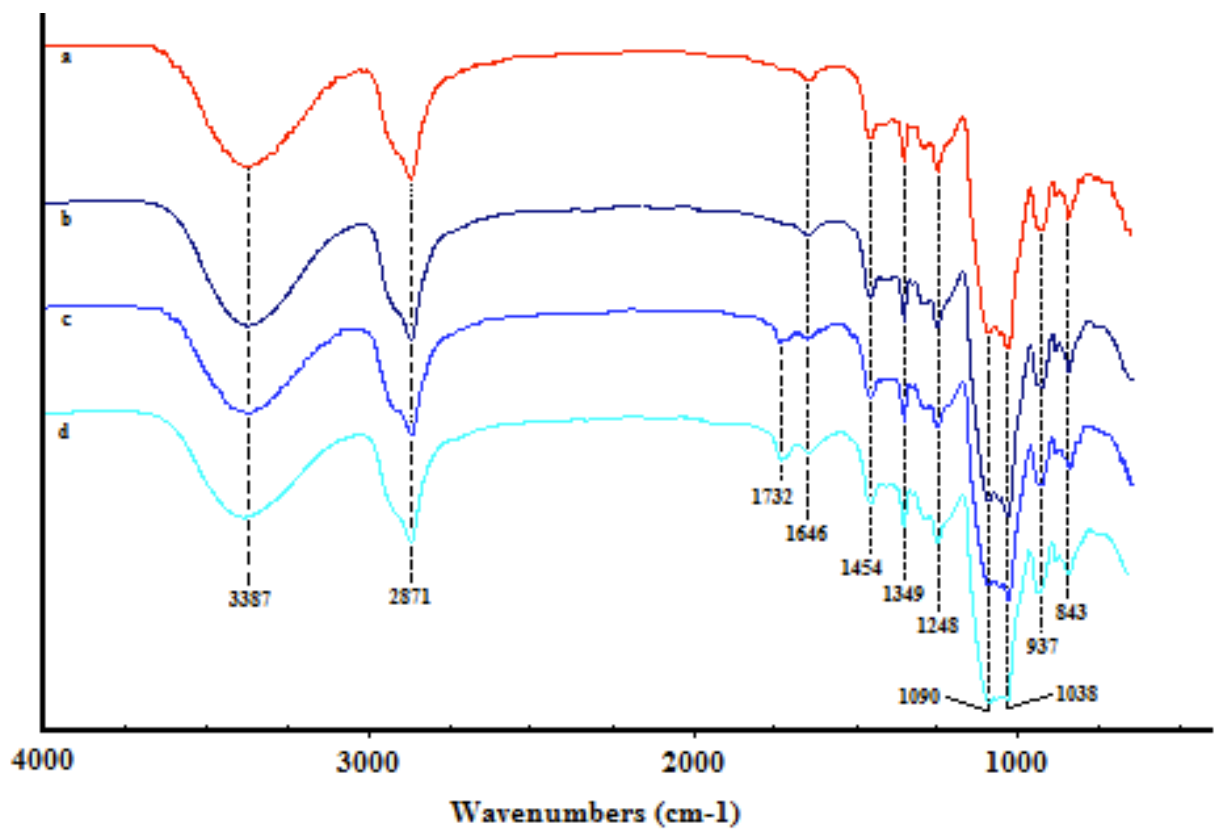

Fig. (6). FTIR spectra of the liquefied biomass-based polyols of wood under conventional bath heating. a. 5 min; b. 30 min; c. 60 min; d. 120 min. (Conventional bath heating conditions: PEG 400/glycerin=7/3, sulfuric acid concentration=3\%, solvent/wood weight ratio=7, $\left.150^{\circ} \mathrm{C}\right)$. 
Table 2. Foam Formulations for the Liquefied Biomass-Based Polyols

\begin{tabular}{|c|c|c|}
\hline & Ingredients & Parts by weight \\
\hline \hline \multirow{2}{*}{ A liquid: } & Biomass-based polyol (12.5\% biomass con.) & 100 \\
& Catalyst (DBTDL) & 1 to 1.5 \\
& Surfactant (SH-193) & 2 to 2.5 \\
& Blowing agent (water, including the water from Neutralization with NaOH solution) & 2 to 4.25 \\
& Additives (PEG \#400) & 15 \\
\hline B liquid: & MDI & 100 to 240 \\
& (isocyanate index) & 80 to 120 \\
\hline
\end{tabular}

efficiency and the foamability of the ingredients, to add a small amount of an appropriate PEG \#400 and use an organic tin catalyst, dibutyltin dilaurate (DBTDL), instead of triethylene diamine, are effective for the foaming. The characteristics of the foams obtained at different isocyanate index are shown in Table $\mathbf{3}$.

From Table 3, compared to fossil-based polyols (especially mixture of PEG \#400 and glycerin), the foam made from liquefied biomass-based polyols under microwave has the lower compressive strength and elastic modulus. These results suggest that liquefied wood mixture can replace part of polyols for the preparation of polyurethane foam, but that will cause the compressive strength and modulus loss. However, the foams made from fossil-based polyols show more brittle than those made from liquefied biomass-based polyols. The permanent deformation of the foams after compression measurements (20\% strain) are more than $7 \%$ for the foams made from fossil-based polyols. Whereas, the permanent deformation of the foams made from biomass-based polyols are less 3\%. These results suggest that the introduction of wood evidently results in the foam with greater resilience. Some of the liquefied wood components can make contributions to the resilience of the resulting foams. However, the foam made form the liquefied biomass-based polyols under the conventional bath heating showed a better compressive strength and elastic modulus, due to a higher hydroxyl number.

With the microwave-assistant liquefaction time increases, the foams made from the liquefied biomass-based polyols obtained show lower mechanical properties. That is attributed to the lower hydroxyl value at long liquefaction time. The compressive strength and apparent modulus increase at isocyanate index from 80 to 100 , then decrease at isocyanate index from 100 to 120 , except the liquefaction

Table 3. Mechanical Properties of the Foams

\begin{tabular}{|c|c|c|c|c|c|}
\hline Reaction Time [min] & $\begin{array}{l}\text { Isocyanate } \\
\text { Index }\end{array}$ & $\begin{array}{l}\text { Density } \\
{\left[\mathrm{g} / \mathrm{cm}^{3}\right]}\end{array}$ & $\begin{array}{l}\text { Compressive } \\
\text { Strength [Kpa] }\end{array}$ & $\begin{array}{c}\text { Apparent } \\
\text { Modulus [Mpa] }\end{array}$ & $\begin{array}{c}\text { Permanent } \\
\text { Deformation }^{\text {a) }}[\%]\end{array}$ \\
\hline 5 & $\begin{array}{c}80 \\
100 \\
120\end{array}$ & $\begin{array}{l}0.035 \\
0.035 \\
0.035\end{array}$ & $\begin{array}{c}8.04 \\
69.92 \\
105.88\end{array}$ & $\begin{array}{l}0.21 \\
2.25 \\
2.93\end{array}$ & $\begin{array}{l}0.63 \\
1.36 \\
1.46\end{array}$ \\
\hline 30 & $\begin{array}{c}80 \\
100 \\
120\end{array}$ & $\begin{array}{l}0.034 \\
0.037 \\
0.035\end{array}$ & $\begin{array}{c}6.68 \\
61.00 \\
90.08\end{array}$ & $\begin{array}{l}0.16 \\
1.35 \\
2.47\end{array}$ & $\begin{array}{l}0.83 \\
1.46 \\
2.91\end{array}$ \\
\hline 60 & $\begin{array}{c}80 \\
100 \\
120\end{array}$ & $\begin{array}{l}0.036 \\
0.035 \\
0.035\end{array}$ & $\begin{array}{c}8.13 \\
55.54 \\
85.84\end{array}$ & $\begin{array}{l}0.14 \\
0.91 \\
1.86\end{array}$ & $\begin{array}{l}1.02 \\
1.24 \\
1.99\end{array}$ \\
\hline 120 & $\begin{array}{c}80 \\
100 \\
120\end{array}$ & $\begin{array}{l}0.036 \\
0.037 \\
0.035\end{array}$ & $\begin{array}{c}8.76 \\
49.54 \\
69.65\end{array}$ & $\begin{array}{l}0.22 \\
1.35 \\
1.66\end{array}$ & $\begin{array}{l}1.13 \\
1.59 \\
1.79\end{array}$ \\
\hline 120 (conventional bath heating) & $\begin{array}{c}80 \\
100 \\
120\end{array}$ & $\begin{array}{l}0.035 \\
0.035 \\
0.035\end{array}$ & $\begin{array}{c}14.05 \\
81.83 \\
140.60\end{array}$ & $\begin{array}{l}1.14 \\
2.05 \\
3.35\end{array}$ & $\begin{array}{l}1.26 \\
1.32 \\
1.46\end{array}$ \\
\hline PEG \#400 & $\begin{array}{c}80 \\
100 \\
120\end{array}$ & $\begin{array}{c}0.035 \\
0.035 \\
-\end{array}$ & $\begin{array}{c}7.65 \\
52.44 \\
-\end{array}$ & $\begin{array}{c}0.16 \\
1.21 \\
-\end{array}$ & $\begin{array}{c}1.92 \\
2.14 \\
-\end{array}$ \\
\hline mixture of PEG \#400 \& glycerin $(70 / 30, w / w)$ & $\begin{array}{c}80 \\
100 \\
120\end{array}$ & $\begin{array}{l}0.034 \\
0.034 \\
0.037\end{array}$ & $\begin{array}{c}88.28 \\
95.62 \\
102.53\end{array}$ & $\begin{array}{l}2.78 \\
2.48 \\
2.18\end{array}$ & $\begin{array}{c}7.12 \\
10.01 \\
9.10\end{array}$ \\
\hline
\end{tabular}

${ }^{\mathrm{a})}$ The permanent deformations of the foams after compression tests ( $20 \%$ strain). 
time of $30 \mathrm{~min}$. These results are attributed to the lower density of the foam at isocyanate index 120 , and the foam cells are bigger than those of the foam at isocyanate index 80 and 120. From Table 3, the foams made from the liquefied biomass-based polyols at 5 min of liquefaction time show the best mechanical properties at isocyanate index 120 .

\section{CONCLUSION}

Comparing to the conventional bath heating, the rapid liquefaction within $2 \mathrm{~min}$ of wood in the presence of PEG 400 /glycerin by using microwave as the heating source was acheived. The liquefaction conditions, such as liquefaction time, temperature, solvent/wood weight ratio, moisture content of wood, and sulfuric acid catalyst concentration influenced the liquefaction and characteristics of the liquefied biomass-based polyols. It was found that acceptable polyols for preparation of rigid polyurethane foams could be obtained by the microwave-assistant rapid liquefaction of wood. The liquefied biomass-based polyols are uniform black liquid with no wood solid residue, 1.13 $\mathrm{g} / \mathrm{cm} 3$ of the specific gravity, the apparent viscosity in the range of 1420 to $1720 \mathrm{cP}$ and the hydroxyl value in range of 290 to $360 \mathrm{mg} \mathrm{KOH/g}$. The biomass-based polyols are suitable for preparation of rigid polyurethane foams. The foams made from the biomass-based polyols obtained at 5 min of liquefaction time show the best mechanical properties at isocyanate index 120 .

\section{ACKNOWLEDGEMENTS}

We are grateful for the financial supports of this research National Natural Science Foundation of China (No.30800867), Program for New Century Excellent Talents in University (No.NCET-09-0906), Key Projects of Chinese Ministry of Education (No. 209117), and Science \& technology funds of Yunnan province, China (No.2007C068M). We are also grateful for the supports of Key Discipline on Wood Science \& Technology in Yunnan Province, China.

\section{REFERENCES}

[1] Wilson JE, Fowler RH. Rigid urethane foams based on sorbitol derivatives. Science 1958; $128: 1343$.

[2] Wismer M, Gibsonia, Foote JF. Method of preparing polyethers of mono and disaccharides. US3085085, April 9, 1963.

[3] Hostettler F, Barnes RK, Mclaughlin RW. Polyurethane foams. US3073788, January 17, 1963.

[4] Allan GG. Hydroxyesters of bark phenolic acids. US3476795, November 4, 1969.

[5] Wu LC-F, Glasser WG. Engineering plastics from lignin. Synthesis of hydroxypropyl lignin. J Appl Polym Sci 1984; 29: 1111-23.

[6] Hostettler F. Polyurethane foams containing stabilized amylaceous materials. US4156759, May 29, 1979.

[7] Yoshida H, Mörck R, Kringstad KP, Hatakeyama H. Kraft lignin in polyurethanes. Mechanical properties of polyurethanes from a kraft lignin-polyether triol-polymeric MDI system. J Appl Polym Sci 1987; 34: 1187-98

[8] Cunningham RL, Carr ME, Bagley EB. Preparation and properties of rigid polyurethane foams containing modified cornstarches. J Appl Polym Sci 1992; 44: 1477-83.

[9] Shiraishi N, Onodera S, Ohtani M, Masumoto T. Dissolution of etherified of esterified wood into polyhydric alcohols or bisphenol A and their application in preparing wooden polymeric materials. Mokuzai Gakkaishi 1985; 31: 418-20.

[10] Yao Y, Yoshioka M, Shiraishi N. Combined liquefaction of wood and starch in a polyethylene glycol/glycerin blended solvent. Mokuzai Gakkaishi 1993; 39: 930-38.

[11] Yao Y, Yoshioka M, Shiraishi N. Rigid polyurethane foams from combined liquefied mixtures of wood and starch. Mokuzai Gakkaishi 1995; 41: 659-68.

[12] Lee SH, Yoshioka M, Shiraishi N. Liquefaction of corn bran (CB) in the presence of alcohols and preparation of polyurethane foam from its liquefied polyol. J Appl Polym Sci 2000; 78: 319-25.

[13] Ge J, Zhang Z, Xu J. Studies on the biodegradable polyurethane materials based on bagasse (I) The liquefaction of bagasse and preparation of polyether ester polyol. Polym Mater Sci Eng 2003; 19: 194-98. (In Chinese)

[14] Wang H, Chen H-Z. A novel method of utilizing the biomass resource: Rapid liquefaction of wheat straw and preparation of biodegradable polyurethane foam (PUF). J Chin Inst Chem Eng 2007; 38: 95-102.

[15] Kurimoto Y, Doi S, Tamura Y. Species effects on woodliquefaction in polyhydric alcohols. Holzforschung 1999; 53: 61722. 